\title{
Crystal structure of tris(tetramethylammonium) dibismuth nonaiodide, $\left[\mathbf{N}\left(\mathrm{CH}_{3}\right)_{4}\right]_{3} \mathbf{B i}_{2} \mathbf{I}_{9}$
}

\author{
C. Feldmann* \\ Philips GmbH Forschungslaboratorien. Weisshausstraße 2. D-52066 Aachen, Germany
}

Received March 23. 2001, CCDC-No. $1267 / 644$

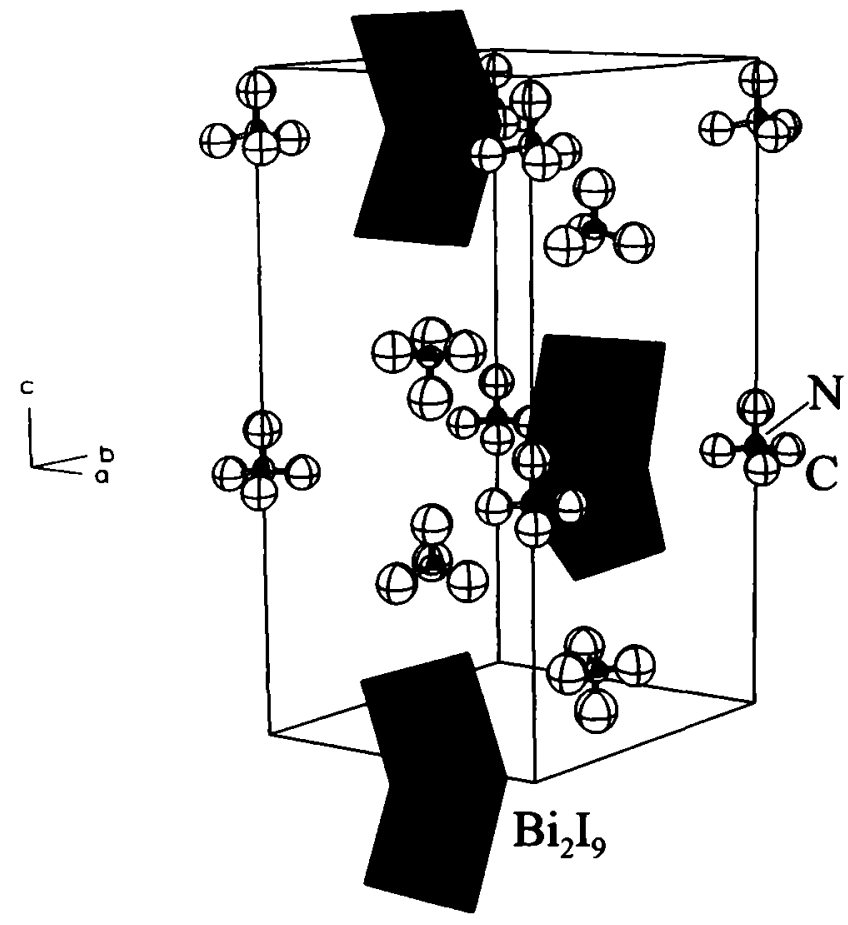

Experimental details

The hydrogen atoms were not localized.

\section{Discussion}

The crystal structure of $\left[\mathrm{N}\left(\mathrm{CH}_{3}\right)_{4}\right]_{3} \mathrm{Bi}_{2} \mathrm{Ig}$ is characterized by two face-sharing $\mathrm{BiI}_{6}$ octahedra forming dimeric $\left[\mathrm{Bi}_{2} \mathrm{I}_{9}\right]^{3-}$ anions as in $\mathrm{Cs}_{3} \mathrm{Bi}_{2} \mathrm{Ig}[2,3]$. Bond length are $d(\mathrm{Bi}-\mathrm{I})_{\text {endo }}=3.27(1) \AA$ and $d(\mathrm{Bi}-\mathrm{I})_{\text {exo }}=2.95(1) \AA$. The unidirectional orientation of the tetramethylammonium cations causes a symmetry reduction compared to $\mathrm{Cs}_{3} \mathrm{Bi}_{2} \mathrm{I}_{9}\left(P 6_{3} / m m c\right)$. Bond lengths and angles in the cation are: $d(\mathrm{~N}-\mathrm{C})=1.45 \AA-1.47(2) \AA, \angle \mathrm{C}-\mathrm{N}-\mathrm{C}=104^{\circ}-$ $114(2)^{\circ}$.

Table 1. Data collection and handling.

$\begin{array}{ll}\text { Crystal: } & \begin{array}{l}\text { orange hexagonal plate, } \\ \text { size } 0.08 \times 0.20 \times 0.20 \mathrm{~mm}\end{array} \\ & \text { Mo } K_{\alpha} \text { radiation }(0.71073 \AA) \\ \text { Wavelength: } & 158.08 \mathrm{~cm}^{-1} \\ \mu: & \text { Siemens P4, } \omega \\ \text { Diffractometer, scan mode: } & 44.96^{\circ} \\ 2 \theta_{\text {max }}: & 13041,1755 \\ N(h k l)_{\text {measured, }} N(h k l)_{\text {unique: }}: & I_{\text {obs }}>2 \sigma\left(I_{\text {obs }}\right), 1556 \\ \text { Criterion for } I_{\text {obss }} N(h k l)_{g t}: & 62 \\ N(\text { param })_{\text {refined: }} & \text { SHELXS-97 [4], SHELXL-97 [5] } \\ \text { Programs: } & \end{array}$

\section{Abstract}

$\mathrm{C}_{12} \mathrm{H}_{36} \mathrm{Bi}_{2} \mathrm{I}_{9} \mathrm{~N}_{3}$, trigonal, $P 31 c$ (No. 159), $a=9.908(1) \AA$, $c=23.420(1) \AA, V=1991.1 \AA^{3}, Z=2, R_{\mathrm{gt}}(F)=0.036$, $w R_{\text {ref }}\left(F^{2}\right)=0.112, T=293 \mathrm{~K}$.

\section{Source of material}

The title compound was obtained by reaction of equimolar amounts of $\left[\mathrm{N}\left(\mathrm{CH}_{3}\right)_{4}\right] \mathrm{I}$ and $\mathrm{BiI}_{3}$ in diethylene glycol at $413 \mathrm{~K}$ for 2 days [1].

Programs:

SHELXS-97 [4], SHELXL-97 [5]

Table 2. Atomic coordinates and displacement parameters (in $\AA^{2}$ ).

\begin{tabular}{llllll}
\hline Atom & Site & $x$ & $y$ & $z$ & $U_{\text {iso }}$ \\
\hline $\mathrm{C}(1)$ & $6 c$ & $0.485(4)$ & $-0.203(6)$ & $-0.082(2)$ & $0.24(2)$ \\
$\mathrm{C}(2)$ & $2 b$ & $1 / 3$ & $2 / 3$ & $-0.003(2)$ & $0.28(6)$ \\
$\mathrm{C}(3)$ & $6 c$ & $0.798(6)$ & $0.481(5)$ & $0.770(2)$ & $0.27(3)$ \\
$\mathrm{C}(4)$ & $2 b$ & $2 / 3$ & $1 / 3$ & $0.691(1)$ & $0.29(3)$ \\
$\mathrm{C}(5)$ & $6 c$ & $1.131(4)$ & $-0.022(5)$ & $0.109(1)$ & $0.19(2)$ \\
$\mathrm{C}(6)$ & $2 a$ & 0 & 0 & $0.032(1)$ & $0.18(1)$
\end{tabular}

Table 3. Atomic coordinates and displacement parameters (in $\AA^{2}$ ).

\begin{tabular}{|c|c|c|c|c|c|c|c|c|c|c|}
\hline Atom & Site & $x$ & $y$ & $z$ & $U_{11}$ & $U_{22}$ & $U_{33}$ & $U_{12}$ & $U_{13}$ & $U_{23}$ \\
\hline $\mathrm{Bi}(1)$ & $2 b$ & $2 / 3$ & $1 / 3$ & $0.1830(1)$ & $0.067(1)$ & $U_{11}$ & $0.050(1)$ & $U_{11} / 2$ & 0 & 0 \\
\hline $\mathrm{Bi}(2)$ & $2 b$ & $2 / 3$ & $1 / 3$ & $-0.0004(1)$ & $0.067(1)$ & $U_{11}$ & $0.050(1)$ & $U_{11} / 2$ & 0 & 0 \\
\hline $\mathrm{I}(1)$ & $6 c$ & $0.5408(2)$ & $0.0462(1)$ & $0.0912(1)$ & $0.081(1)$ & $0.058(1)$ & $0.070(1)$ & $0.031(1)$ & $0.000(1)$ & $0.000(1)$ \\
\hline $\mathrm{I}(2)$ & $6 c$ & $0.3782(2)$ & $0.1695(2)$ & $0.9319(1)$ & $0.084(1)$ & $0.102(1)$ & $0.084(1)$ & $0.038(1)$ & $-0.026(1)$ & $-0.011(1)$ \\
\hline $\mathrm{I}(3)$ & $6 c$ & $0.3782(2)$ & $0.1693(2)$ & $0.2507(1)$ & $0.086(1)$ & $0.102(1)$ & $0.083(1)$ & $0.038(1)$ & $0.025(1)$ & $0.011(1)$ \\
\hline$N(1)$ & $2 b$ & $1 / 3$ & $2 / 3$ & $-0.066(1)$ & $0.06(1)$ & $U_{11}$ & $0.06(2)$ & $U_{\mathrm{I}} / 2$ & 0 & 0 \\
\hline$N(2)$ & $2 b$ & $2 / 3$ & $1 / 3$ & $0.753(1)$ & $0.07(1)$ & $U_{11}$ & $0.03(1)$ & $U_{11} / 2$ & 0 & 0 \\
\hline$N(3)$ & $2 a$ & 0 & 0 & $0.094(1)$ & $0.05(1)$ & $U_{11}$ & $0.07(1)$ & $U_{11} / 2$ & 0 & 0 \\
\hline
\end{tabular}

* (e-mail: claus.feldmann@philips.com) 
Acknowledgments. The author is grateful to Prof. Dr. M. Jansen, Stuttgart, for his support as well as to Dr. J. Nuss, Stuttgar, for the data collection.

\section{References}

1. Feldmann, $\mathrm{C} .: \mathrm{CuBi}_{7} \mathrm{I}_{9}\left(\mathrm{C}_{4} \mathrm{H}_{8} \mathrm{O}_{3} \mathrm{H}\right)_{3}\left(\mathrm{C}_{4} \mathrm{H}_{8} \mathrm{O}_{3} \mathrm{H}_{2}\right)$ - a novel complex bismuth iodide containing one-dimensional $\left[\mathrm{CuBi}_{5} \mathrm{I}_{19}\right]^{3-}$ chains. Inorg. Chem. 40 (2001) 818-819.

2. Lindqvist, O.: The Crystal Structure of Cesium Bismuth Iodide, $\mathrm{Cs}_{3} \mathrm{Bi}_{2} \mathrm{I}_{9}$. Acta Chem. Scand. 22 (1968) 2943-2952.

3. Chabot, B.; Parthe, E.: $\mathrm{Cs}_{3} \mathrm{Sb}_{2} \mathrm{Ig}_{9}$ and $\mathrm{Cs}_{3} \mathrm{Bi}_{2} \mathrm{I}_{9}$ with hexagonal $\mathrm{Cr}_{3} \mathrm{Cr}_{2} \mathrm{Cl}_{9}$ structure type. Acta Crystallogr. B34 (1978) 645-648.
4. Sheldrick, G. M.: SHELXS-97, Program for the Solution of Crystal Structures. University of Göttingen, Germany 1997.

5. Sheldrick, G. M.: SHELXL-97, Program for Crystal Structure Refinement. University of Göttingen, Germany 1997. 